\title{
Drosophila, the savvy exterminator
}

\section{Belinda H.TAN ANd Robert L. Modlin}

UCLA School of Medicine,

10833 Le Conte Avenue,

Los Angeles, CA 90095, USA.

rmodlin@mednet.ucla.edu

Recently flies have provided remarkable clues to the mechanisms of mammalian innate immunity, but can we unlock the greater potential of the powerful fly genetic model? Drosophila has evolved a simple but efficient immune system for combating a diverse array of microbial threats. A central mechanism of host defense in Drosophila is mediated by the Toll family of receptors that, upon activation, lead to induction of antimicrobial peptides and hence elimination of foreign invaders. The first clues were unveiled by Medzhitov and Janeway, who identified the mammalian homologs of Drosophila Toll, the Toll-like receptors (TLRs), and demonstrated their participation in innate immunity through induction of cytokines and up-regulation of molecules involved in antigen presentation ${ }^{1}$. We now know that not only is there conservation between fly Toll and human TLR structures, but that striking similarities also exist in downstream components of their respective signaling pathways ${ }^{2}$.

If the immune system of Drosophila can serve as a blueprint for mammalian innate defense, perhaps several new clues warrant closer scrutiny. Recent identification of additional receptors in the Drosophila Toll family has provided further evidence for specificity in Toll receptor activation: Toll and Toll-5 respond to fungi and Gram-positive organisms, whereas 18-wheeler and/or perhaps an unidentified receptor mediates the response to Gram-negative organisms ${ }^{3}$. As in Drosophila, different mammalian TLRs confer specificity for distinct classes of microbial ligands ${ }^{4}$.

The class-specific responses of

Drosophila Toll family members to different pathogens provides an efficient mechanism for regulating only those signal transduction pathways and target genes that will lead to the elimination of the particular pathogen ${ }^{5}$. Activation of Toll by fungi leads to a signaling pathway involving the Rel protein DIF and induction of Drosomycin, an antimicrobial peptide with activity against fungi but not bacteria ${ }^{6}$. In contrast, neither
Toll nor DIF is required for Gram-negative organisms to induce a matrix of antibacterial peptides such as diptericin, cecropin, defensin and attacin. Rather, these peptide are regulated by a separate signal transductor pathway involving a defined IKK, IKK $\gamma$, which regulates the activation of the Rel protein Relish ${ }^{7,8}$.

The presence of mammalian TLRs and associated signaling factors provides evidence supporting a conserved evolutionary response to challenge by infectious agents. Like Drosophila, there is specificity in the response of mammalian TLRs to particular pathogens. Therefore, mammalian TLRs are likely to have conserved, from Drosophila, receptor-specific signaling pathways and downstream targets to provide efficient defense against the appropriate pathogen. The lipopolysaccahride- and interleukin 1 -inducible antimicrobial peptide human $\beta$ defensin 2 may be an example of a conserved defense mechanism similar to the inducible antimicrobial peptides found in Drosophila ${ }^{9}$. Drosophila, the savvy exterminator, provides clues to understanding distinct pathways of mammalian host defense.

Beyond providing clues about innate immunity, Drosophila might serve as an ideal model for studying mammalian immune defense pathways. Creating transgenic flies with mammalian genes is a current technology that makes complementation of mutant receptor pathways both feasible and fashionable. Additionally, modifier genetic screens using these transgenic flies can identify new pathway members that may be responsible for imparting specificity to the functional pathway of interest. Could greater insight into the mechanisms of human TLRs (hTLRs) and their contribution to host defense not be gained by studying transgenic flies with hTLR or hTLR/toll chimeras on a background of Toll family mutations?

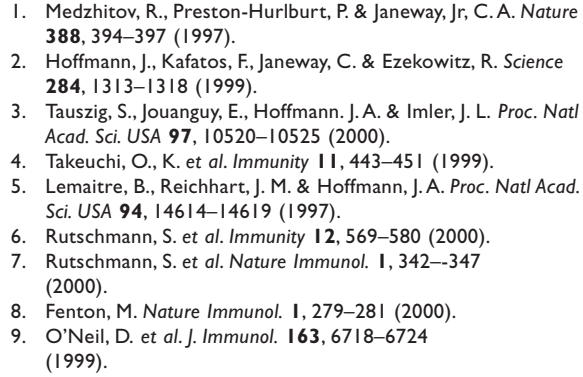

